\section{¿Influye el medio de preservación del injerto en los resultados y costos del trasplante hepático de donante fallecido? Comparación de dos soluciones de preservación}

\author{
JORGE MARTÍNEZ ${ }^{1}$, JEAN P. BÄCHLER ${ }^{1}$, FABRIZIO MOISAN ${ }^{1}$, \\ JAVIERA TORRES ${ }^{2}$, IGNACIO DUARTE ${ }^{2}$, ROSA MARÍA PÉREZ ${ }^{3}$, \\ CARLOS BENÍTEZ ${ }^{3}$, MARCO ARRESE ${ }^{3}$, PILAR DOMÍNGUEZ $Z^{1, a}$, \\ JUAN F. GUERRA ${ }^{3}$, NICOLÁS JARUFE ${ }^{3}$
}

\section{Outcomes using two preservation solutions (UW/HTK) in liver transplantation from brain death donors}

Background: Preservation solutions are critical for organ transplantation. In liver transplant (LT), the solution developed by the University Of Wiscon$\sin (U W)$ is the gold-standard to perfuse deceased brain death donor (DBD) grafts. Histidine-Tryptophan-Ketoglutarate (HTK), formerly a cardioplegic infusion, has been also used in solid organ transplantation. Aim: To compare the outcomes of LT in our center using either HTK or UW solution. Patients and Methods: Retrospective study including 93 LT DBD liver grafts in 89 patients transplanted between March 1994 and July 2010. Forty-eight grafts were preserved with UW and 45 with HTK. Donor and recipient demographics, total infused volume, cold ischemia time, post-reperfusion biopsy, liver function tests, incidence of biliary complications, acute rejection and 12-month graft and patient survival were assessed. Preservation solution costs per liver graft were also recorded. Results: Donor and recipient demographics were similar. When comparing UW and HTK, no differences were observed in cold ischemia time ( $9.6 \pm 3$ and $8.7 \pm 2 \mathrm{~h}$ respectively, $p=0.23$ ), biliary complications, the incidence of acute rejection, primary or delayed graft dysfunction. Histology on post-reperfusion biopsies revealed no differences between groups. The infused volume was significantly higher with HTK than with UW (9 (5-16) and 6 (311) $l, p<0.001$ ). The cost per procurement was remarkably lower using HTK. Conclusions: Perfusion of DBD liver grafts with HTK is clinically equivalent to UW, with a significant cost reduction.

(Rev Med Chile 2014; 142: 1229-1237)

Key words: Cost-benefit analysis; Liver transplantation; Organ preservation solutions.

\author{
Programa de Trasplante Hepático. \\ 'Departamento de Cirugía \\ Digestiva. \\ 2Departamento de Anatomía \\ Patológica. \\ ${ }^{3}$ Departamento de \\ Gastroenterología. \\ Escuela de Medicina. Hospital \\ Clínico Pontificia Universidad \\ Católica de Chile. \\ aEnfermera Universitaria. \\ Fuentes de apoyo financiero: \\ ninguna \\ Los autores no refieren conflictos \\ de interés.

Recibido el 12 de diciembre
de 2013 , aceptado el 10 de
septiembre de 2014.
Correspondencia a:
Dr. Jorge Martínez Castillo
Departamento de Cirugía
Digestiva
Escuela de Medicina. Pontificia
Universidad Católica de Chile.
Marcoleta 367.
Casilla Postal 114-D, Santiago.
Teléfono: 56-2-23543870
jamartin@med.puc.cl \\ jamartin@med.puc.cl
}

4 n los trasplantes de órganos sólidos obtenidos de donantes fallecidos (DF), la inducción de hipotermia y los medios de preservación han sido fundamentales para su éxito ${ }^{1,2}$. Las soluciones de preservación se clasifican en intracelulares y extracelulares de acuerdo a su contenido en potasio, por homología al medio interno o externo de la célula. Entre las primeras, la solución de la Universidad de Wisconsin (UW), desarrollada por Belzer, constituyó un significativo 
avance frente a la anterior solución de Collins ${ }^{1,3}$. En trasplante hepático (TH), la perfusión in-situ del donante con UW ha demostrado resultados óptimos por más de dos décadas, constituyéndose en la solución de preservación estándar ${ }^{2,3}$. La solución extracelular histidina-triptofanoketoglutarato (HTK), inicialmente una solución cardiopléjica $^{4,5}$, se extendió posteriormente a los trasplantes de órganos abdominales ${ }^{6,7}$ por su menor carga de potasio durante la reperfusión ${ }^{6,8,9} \mathrm{y}$ su menor viscosidad ${ }^{10}$.

En nuestro país ambas soluciones se importan, lo que incrementa el costo de los trasplantes en la implementación de la cirugía del donante. Así, nuestro objetivo fue comparar injertos hepáticos de DF preservados con UW o con HTK en TH en nuestro centro.

\section{Pacientes y Método}

Estudio retrospectivo que comparó injertos hepáticos de DF perfundidos con UW con aquellos preservados en HTK, trasplantados en pacientes adultos consecutivos entre marzo de 1994 y julio de 2010. Los componentes de ambas soluciones se especifican en la Tabla $1^{11}$. La asignación de la solución a perfundir fue según disponibilidad al momento de la procura. Entre 1994 y 2002 todos los injertos fueron preservados con UW $(n=26)$. A partir de 2003, con la disponibilidad de HTK, se utilizó ambas soluciones. Un equipo propio realizó la cirugía extractiva en todos los donantes, utilizando perfusión aórtica-portal o aórtica exclusiva indistintamente ${ }^{12,13}$. El volumen infundido fue según recomendación de los proveedores, con el objetivo de "blanquear" los órganos abdominales. La perfusión de ambas soluciones se realizó sólo por gravedad. Se incluyó injertos estándares y de criterios extendidos ${ }^{14}$. La técnica de implantación ${ }^{15}$ y los esquemas inmuno-supresores utilizados en los receptores fueron similares. Se registró y analizó entre otros, datos bio-demográficos de donantes y receptores, de la cirugía extractiva, el volumen perfundido por donante, los tiempos de isquemia, etc. La función del injerto se evaluó por los niveles de aminotransferasas y bilirrubina total (BT) en sangre los días 1, 7, 14 y 30 post-trasplante y mediante la consignación de disfunción inicial o falla primaria de injerto. Se registró las complicaciones biliares, incidencia de rechazo agudo y se analizó las biopsias post-reperfusión. Se calculó la sobrevida de injerto y paciente a 12 meses. Se estimó el costo total de la solución utilizada a partir del valor comercial por litro de cada una de ellas (UW: USD\$ 453/HTK: USD\$ 118).

\section{Definiciones}

Biopsia post-reperfusión: Muestra del hígado implantado tomada posterior a la reperfusión portal y arterial, antes del cierre del abdomen, para análisis histológico diferido. Las alteraciones atribuidas a daño por isquemia-reperfusión se clasificaron histológicamente de la siguiente forma (Figura 1):

- Sin daño: Mínima hiperemia subcapsular, leve infiltrado portal linfocitario, hepatocitos de aspecto normal.

- Daño leve: Leve hiperemia y hemorragia subcapsular, hepatocitos periportales con signos de daño, los cambios no alcanzan la región centro lobulillar.

Tabla 1. Componentes de las soluciones UW y HTK

\begin{tabular}{|c|c|c|}
\hline & UW & HTK \\
\hline Año de Introducción & 1988 & 1975 \\
\hline Autor (Origen) & $\begin{array}{l}\text { F. Belzer } \\
\text { (EEUU) }\end{array}$ & $\begin{array}{l}\text { H. Bretschneider } \\
\text { (Alemania) }\end{array}$ \\
\hline \multicolumn{3}{|l|}{ Componentes } \\
\hline Sodio (mmmol/L) & $25-30$ & 15 \\
\hline Potasio (mmmol/L) & $125-130$ & 10 \\
\hline Magnesio (mmmol/L) & 5 & 13 \\
\hline Calcio (mmmol/L) & - & 0,25 \\
\hline Manitol (g/L) & - & 30 \\
\hline Lactobionato (mmmol/L) & 100 & - \\
\hline HES (g/L) & 50 & - \\
\hline Rafinosa (mmmol/L) & 30 & - \\
\hline Histidina (mmmol/L) & - & 180 \\
\hline $\mathrm{H}_{2} \mathrm{PO}_{4} / \mathrm{HPO}_{4}(\mathrm{mmmol} / \mathrm{L})$ & 25 & - \\
\hline Glutation (mmmol/L) & 3 & - \\
\hline Alopurinol (mmmol/L) & - & 1 \\
\hline Adenosina (mmmol/L) & 5 & - \\
\hline Ketoglutarato (mmmol/L) & - & 1 \\
\hline Triptofano (mmmol/L) & - & 2 \\
\hline $\mathrm{mOsm} / \mathrm{L}$ & 320 & 310 \\
\hline $\mathrm{pH}$ & 7,4 & 7,2 \\
\hline
\end{tabular}



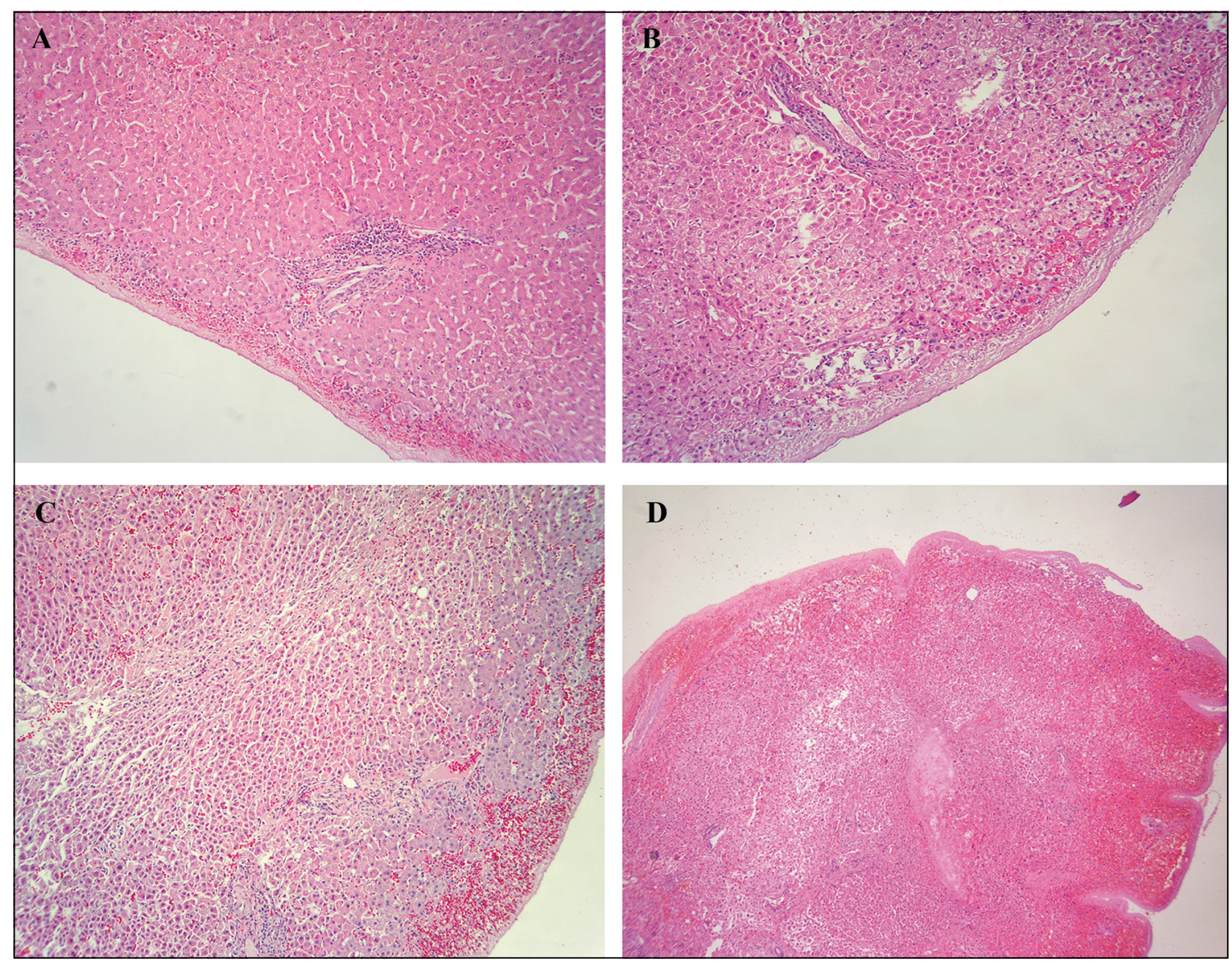

Figura 1. Clasificación del daño histológico en biopsias post-reperfusión de pacientes trasplantados de hígado de donante fallecido. (A) Sin Daño, (B) Daño Leve, (C) Daño Moderado, (D) Daño Severo o Acentuado.

- Daño moderado: Hemorragia e hiperemia subcapsular de mayor extensión con algunos neutrófilos lobulillares, hepatocitos con signos de daño alcanzan la zona centro lobulillar.

- Daño severo o acentuado: Extensa hemorragia e hiperemia subcapsular que alcanza gran parte del parénquima subyacente.

Disfunción precoz del injerto: Condición que cursa con $\mathrm{BT} \geq 10 \mathrm{mg} / \mathrm{dL}$ al día 7 post-trasplante y/o alza de transaminasas $\geq 2.000 \mathrm{U} / \mathrm{L}$ al día 7 post-trasplante ${ }^{16}$.

Falla primaria del injerto: Condición clínica ocurrida dentro de los 10 días posterior al trasplante, de causa no precisada (descartada trombosis de arteria hepática) caracterizada por alza de transaminasas $\geq 5.000 \mathrm{U} / \mathrm{L}$, INR $>3,0$ y acidosis. Esta condición es una emergencia y motivo de re-trasplante o muerte del receptor ${ }^{17}$.

Tiempo de isquemia fría (TIF): Período comprendido entre el inicio de la infusión de la solución fría de preservación en el donante al inicio de la implantación en el receptor (inicio de anastomosis de la vena cava).

Tiempo de isquemia caliente: Período entre el inicio de la anastomosis de la vena cava y la reperfusión portal.

Rechazo (celular) agudo: Evento clínico-patológico sospechado por alteración de las pruebas de función hepática, diagnosticado por biopsia. Sólo se consideraron en este estudio aquellos rechazos agudos que requirieron de tratamiento específico (bolos de esteroides). 


\section{Análisis estadístico}

Las variables fueron expresadas en porcentajes, media y/o mediana según su distribución; ésta evaluada con pruebas de normalidad. Se realizó un análisis univariado entre variables perioperatorias y tipo de solución mediante pruebas de $\chi^{2}$, MannWhitney y T de Student. Se ejecutó un análisis multivariado por regresión logística para testear asociación entre el tipo de solución y disfunción precoz o falla primaria del injerto, complicaciones biliares, rechazo agudo y daño en biopsias postreperfusión. Éste incluyó las variables: edad del receptor y donante, puntaje MELD > 15 y TIF $>12 \mathrm{~h}$. Los resultados se expresaron en Odds Ratio (OR) e intervalo de confianza de 95\% (IC-95\%). Un $\mathrm{p} \leq 0,05$ fue considerado significativo. Se realizaron curvas de sobrevida de injerto y pacientes utilizando Kaplan-Meier y test de Log-rank para establecer diferencias. Se evaluaron variables asociadas a sobrevida mediante un modelo de regresión de Cox, ajustado por edad del donante y receptor, puntaje MELD $>15$ y TIF $>12 \mathrm{~h}$. Los resultados fueron expresados en Hazzard Ratio (HR) e IC-95\%. Se utilizó el programa SPSS 21 para los cálculos estadísticos (IBM SPSS).

\section{Resultados}

Se realizaron 114 trasplantes en 110 pacientes en el período. En 99, se realizó biopsia hepática post-reperfusión; en 3, no se registró el medio de preservación utilizado; en 2, se utilizó una mezcla de soluciones y en 1 se perfundió solución EuroCollins. Así, la serie comprendió el análisis de 93 injertos: 48 preservados con UW y 45 perfundidos con HTK; los cuales fueron trasplantados en 89 pacientes (45, recibieron injertos preservados con UW y 44, con HTK). Las características biodemográficas y quirúrgicas de los 93 donantes/ injertos se resumen en la Tabla 2. En ella destaca que los hígados perfundidos con UW fueron de donantes más jóvenes y que su volumen infundido fue menor (UW: 6 L [3-11] versus HTK: 9 L [516], $\mathrm{p}<0,001)$. No se observó otras diferencias significativas entre los grupos, si bien el TIF de los hígados preservados con UW pareció discretamente mayor $(p=0,23)$. En la mayoría de las procuras se perfundió por vía aórtica exclusiva (UW: 60\% y HTK: 69\%).

Las variables bio-demográficas y quirúrgicas de los receptores se resumen en la Tabla 3. Hubo 4 pacientes re-trasplantados, 2 por rechazo crónico, otro por trombosis de arteria hepática y el cuarto por trombosis de las venas suprahepáticas y porta. De los 4 injertos implantados inicialmente, 2 habían sido perfundidos con UW y 2 con HTK. En tanto, al momento del re-trasplante, 3 injertos fueron perfundidos con UW y sólo uno con HTK. No hubo diferencias significativas entre ambos grupos, excepto en la cantidad de glóbulos rojos transfundidos durante el trasplante, que fue significativamente mayor en los pacientes con hígado preservado en UW.

Tabla 2. Características del donante e injerto

\begin{tabular}{|c|c|c|c|}
\hline & $\begin{array}{c}\text { Grupo UW } \\
\text { ( }=48 \text { Inj/45 Pctes) }\end{array}$ & $\begin{array}{c}\text { Grupo HTK } \\
\text { (n = } 45 \text { Inj/44 Pctes) }\end{array}$ & Valor $\mathbf{p}$ \\
\hline Edad Donante (años) & $38 \pm 15$ & $41 \pm 14$ & 0,05 \\
\hline Sexo (hombre) & $34(71 \%)$ & $25(56 \%)$ & \\
\hline Esteatosis $\geq 60 \% \S$ & $6(13 \%)$ & $10(22 \%)$ & 0,28 \\
\hline Tiempo de Isquemia Fría (h) & $9,6 \pm 3$ & $8,7 \pm 2$ & 0,23 \\
\hline Tiempo de Isquemia Caliente (min) & $38(30-46)$ & $36(30-44)$ & 0,13 \\
\hline Injerto de Criterio Extendido & $22(46 \%)$ & $25(56 \%)$ & 0,35 \\
\hline Perfusión Ao exclusiva/Ao-P & $29(60 \%) / 19(40 \%)$ & $31(69 \%) / 14(31 \%)$ & 0,33 \\
\hline Total de solución utilizada $(\mathrm{L})^{\S \S}$ & $6(3-11)$ & $9(5-16)$ & $<0,001$ \\
\hline Costos de solución utilizada (USD\$) & $2.722(1.359-4.983)$ & $1.062(590-1.888)$ & $<0,01$ \\
\hline
\end{tabular}

*Variables cuantitativas expresados en mediana y rango, media \pm desviación estándar según su distribución. Ao: Aórtica, Ao-P: Aórtico-portal, Inj: injerto, Pctes: pacientes. ${ }^{\S}$ Porcentaje de esteatosis en biopsia de órgano donante. ${ }^{\S} \mid$ Incluye cirugía de banco. 
Soluciones de preservación en trasplante hepático - J. Martínez et al

Tabla 3. Características del receptor

\begin{tabular}{|c|c|c|c|}
\hline & $\begin{array}{c}\text { Grupo UW } \\
\text { ( }=48 \text { Inj/45 Pctes) }\end{array}$ & $\begin{array}{c}\text { Grupo HTK } \\
\text { (n = } 45 \text { Inj/44 Pctes) }\end{array}$ & Valor $\mathbf{p}$ \\
\hline Edad Receptor (años) & $53(44-62)$ & $60 \quad(49-63)$ & 0,03 \\
\hline Sexo (hombre) & $21 \quad(44 \%)$ & $22 \quad(46 \%)$ & NS \\
\hline Re-trasplante & $3(6,25 \%)$ & $1(2,22 \%)$ & \\
\hline \multicolumn{4}{|l|}{ Diagnóstico de base } \\
\hline $\mathrm{HCC}^{* *}$ & $6 \quad(13 \%)$ & $10 \quad(11 \%)$ & NS \\
\hline Alcohol & $4 \quad(8 \%)$ & $(22 \%)$ & NS \\
\hline Autoinmune & $(17 \%)$ & $(16 \%)$ & NS \\
\hline $\mathrm{HCV}$ & $11 \quad(23 \%)$ & $(13 \%)$ & NS \\
\hline $\mathrm{NASH}$ & $(13 \%)$ & $(11 \%)$ & NS \\
\hline Criptogénica & $(10 \%)$ & $(9 \%)$ & NS \\
\hline Hemocromatosis & $5 \quad(10 \%)$ & $(13 \%)$ & NS \\
\hline Otros & $(13 \%)$ & $(9 \%)$ & NS \\
\hline $\mathrm{FHF}$ & $3 \quad(6 \%)$ & $(7 \%)$ & NS \\
\hline MELD previo al TH & $20 \quad(15-27)$ & $16(12-21)$ & NS \\
\hline Transfusión GR (unidades) & $(2-8)$ & $(2-5)$ & 0,02 \\
\hline
\end{tabular}

*Variables cuantitativas expresados en mediana y rango, media \pm desviación estándar según su distribución. TH: trasplante hepático, GR: glóbulos rojos, HCV: virus hepatitis C, NASH: esteatohepatitis no alcohólica, DHC: daño hepático crónico, HCC: Hepatocarcinoma, MELD: Model for End-stage Liver Disease, FHF: falla hepática fulminante, Inj: injerto, Pctes: pacientes, NS: no significativo. **Pacientes incluidos dentro del grupo con DHC.

Tabla 4. Hallazgos histológicos en biopsias post-reperfusión según tipo de solución utilizada

\begin{tabular}{|lccc|}
\hline Hallazgos histológicos & $\begin{array}{c}\text { Grupo U.w } \\
\text { (n= 48Inj/45 Pctes) }\end{array}$ & $\begin{array}{c}\text { Grupo HTK } \\
\text { (n = 45Inj/44 Pctes) }\end{array}$ & Valor p \\
\hline Sin Daño & $19(39,6 \%)$ & $15(33,3 \%)$ & 0,68 \\
Daño Leve & $19(39,6 \%)$ & $21(46,7 \%)$ & 0,63 \\
Daño Moderado & $5(10,4 \%)$ & $8(17,8 \%)$ & 0,46 \\
Daño severo o acentuado & $5(10,4 \%)$ & $1(2,2 \%)$ & 0,23 \\
\hline
\end{tabular}

La Figura 2 muestra la evolución de los niveles de alanino y aspartato aminotransferasas (ALT y AST) y BT los días 1, 7, 14 y 30 post-trasplante, según el tipo de solución utilizada. Los niveles de AST en día 1 fueron mayores con HTK $(p=0,043)$; en cambio, la BT fue significativamente menor $(\mathrm{p}=0,032)$. En los días siguientes no se observó diferencias significativas en las pruebas de función hepática. Se consignó una disfunción inicial del injerto en 7 órganos (15\%) perfundidos con UW y en $10(22,2 \%)$ con HTK $(\mathrm{p}=0,49)$ y una falla primaria con cada una de las soluciones. Estos 2 pacientes no lograron ser re-trasplantados $\mathrm{y}$ fallecieron.
Se registraron complicaciones biliares en 17 injertos (35\%) preservados con UW y en 15 (33\%) con HTK $(\mathrm{p}=0,99)$, siendo la más frecuente la estenosis de la anastomosis bilio-biliar. Doce injertos (25\%) perfundidos con UW y 7 (16\%) con HTK presentaron un rechazo agudo $(\mathrm{p}=0,27)$.

La Tabla 4 describe los hallazgos histológicos de las biopsias post-reperfusión. Cerca de $80 \%$ en ambos grupos presentó daño leve o ausencia de daño post-reperfusión, no observándose diferencias significativas en cada una de las categorías analizadas.

La Tabla 5 muestra el análisis uni y multivariado entre el tipo de solución utilizada y los 

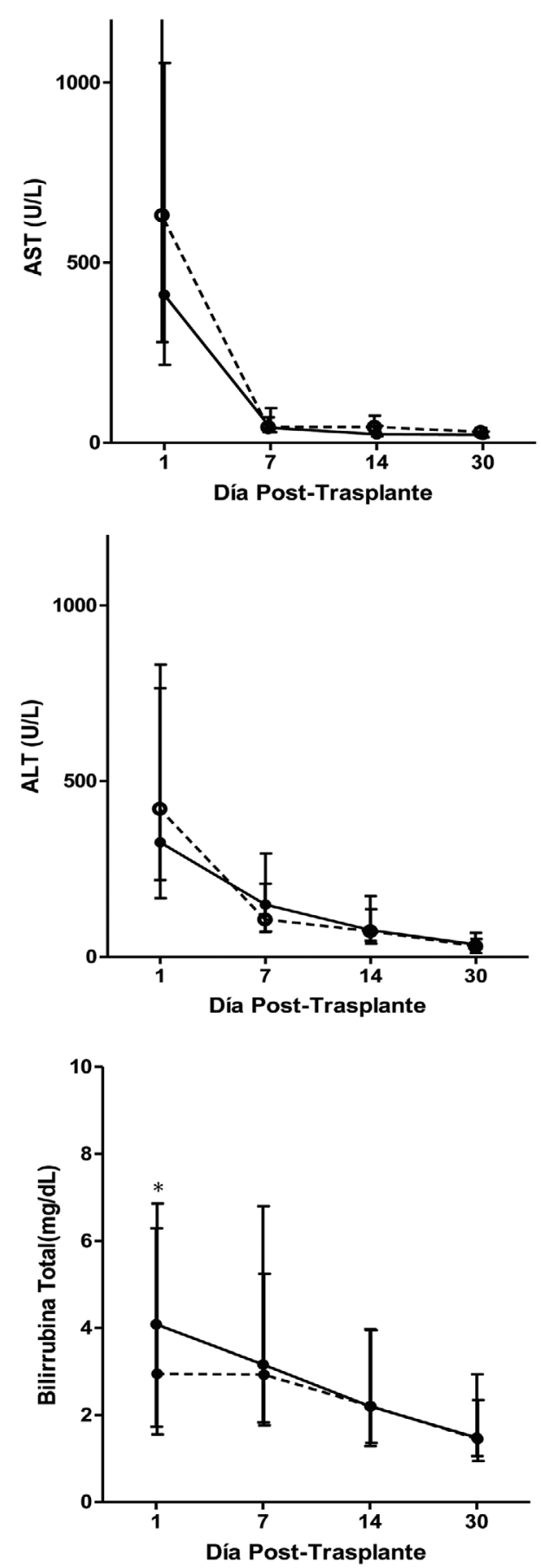

Figura 2. Evolución de los niveles de aminotransferasas AST, ALT y bilirrubina total al día 1, 7, 14 y 30 post-trasplante hepático según el tipo de solución de preservación utilizada (UW: línea continua/HTK: línea discontinua). ${ }^{*} p<0,05$. resultados perioperatorios e histológicos. No se observó asociación entre la solución empleada y la incidencia de disfunción inicial o falla primaria del injerto, complicaciones biliares, rechazo agudo, ni daño post-reperfusión. Lo anterior se mantuvo posterior al ajuste por confundentes.

La Figura 3 muestra las curvas de sobrevida de los injertos según solución de preservación; 78\% y $76 \%$ a los 12 meses para UW y HTK respectivamente, sin diferencias significativas entre ellas. Tampoco existió diferencias en la sobrevida de los pacientes. El análisis univariado no muestra asociación entre la solución utilizada y la sobrevida del injerto (HR: 1,1 IC 95\%: 0,4-2,5) lo que se mantiene en el análisis multivariado (HR: 1,6 IC 95\%: 0,5-5,6).

Los costos por donante según solución de preservación utilizada, fueron significativamente mayores cuando se utilizó UW (USD\$ 2.722 [1.359-4.983]) en relación a HTK (USD\$ 1.062 [590-1.888]. $\mathrm{p}<0,01)$.

\section{Discusión}

Esta es la primera comunicación nacional que compara los resultados en $\mathrm{TH}$ utilizando 2 soluciones de preservación: UW versus HTK. Esta información es relevante en nuestro medio, dada la restricción de recursos asignados a los trasplantes de órganos y a que la implementación de programas de trasplante debe considerar la importación de soluciones de preservación, su eficacia y los costos involucrados en el procedimiento.

Posterior al desarrollo de la preservación en frío y de las primeras soluciones intracelulares de preservación, el advenimiento de la solución UW propiciada por Belzer permitió la expansión de los trasplantes de órganos especialmente extrarrenales, dada la posibilidad de mantenerlos viables por tiempos de isquemia prolongados ${ }^{3}$. A partir de la década 1990-99, los principales programas de $\mathrm{TH}$ nacionales comenzaron con UW en la procura multiorgánica ${ }^{18,19}$. Nuestro grupo decidió realizar el presente estudio, considerando que el costo de la cirugía del donante podría ser reducido utilizando una solución de preservación alternativa, como es HTK, con datos en la literatura que apoyaban su uso en trasplante de órganos sólidos y que incluso mostraban beneficios en comparación a UW $\mathrm{UW}^{9,11}$.

En este análisis comparativo, dos grupos de 
Soluciones de preservación en trasplante hepático - J. Martínez et al

Tabla 5. Análisis de resultados perioperatorios según solución de perfusión utilizada

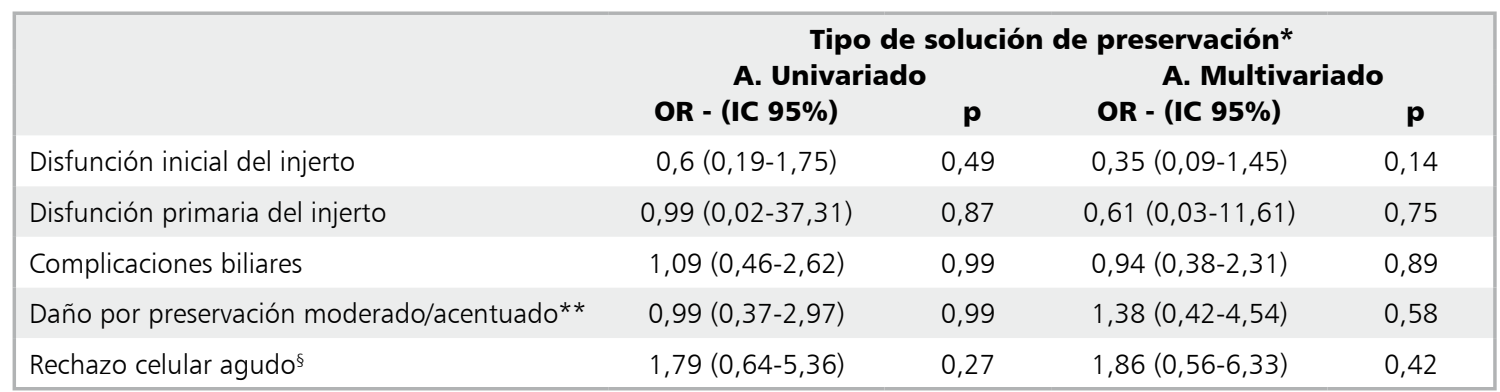

*Solución de referencia: Universidad de Wisconsin. ${ }^{*}$ En biopsias de injerto post-reperfusión. ${ }^{* * *}$ Resultados expresados en Odds Ratio (OR) e Intervalos de Confianza 95\% (IC 95\%) Prueba de Hosmer y Lemeshow p > 0,5 para cada uno de los modelos. $\S$ Incluye sólo a aquellos pacientes que recibieron tratamiento específico para esta condición

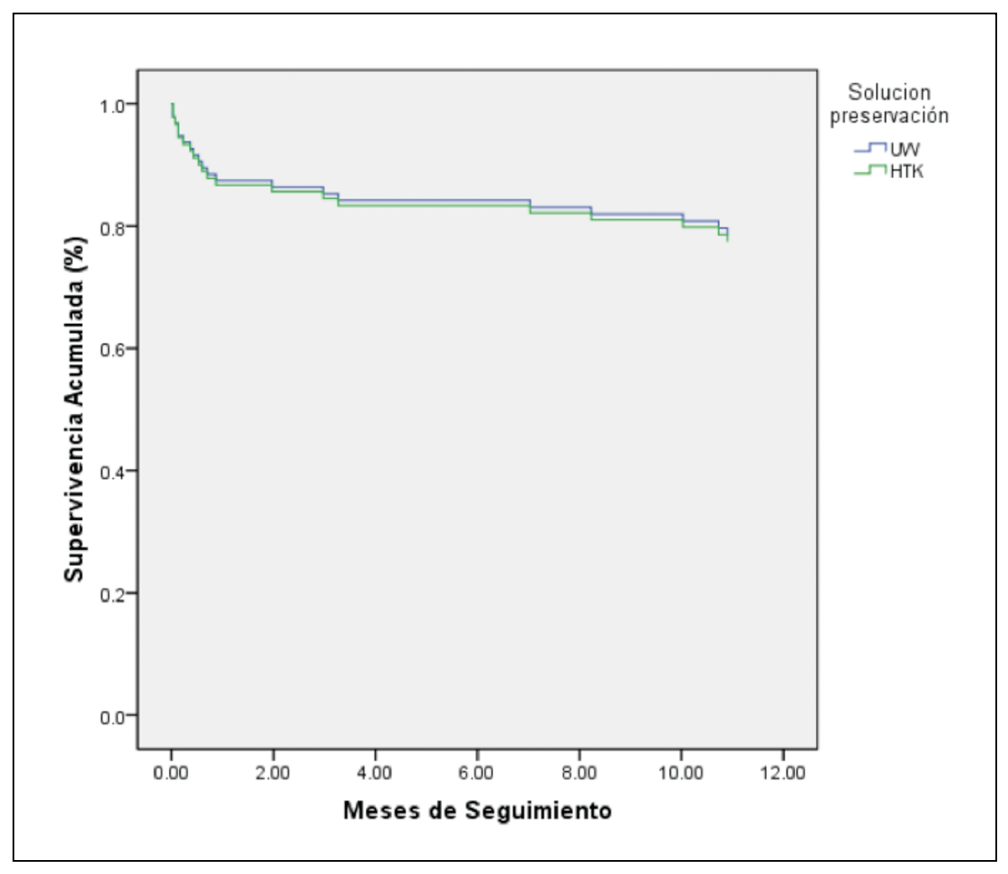

Figura 3. Sobrevida de los injertos preservados con UW $(n=48)$ y HTK $(n=45)$. No existe diferencia significativa entre ambas curvas. Test de Long-rank $p=0,32$. injertos preservados con alguna de las soluciones evaluadas, trasplantados en pacientes adultos -del todo similares con respecto a características biodemográficas y clínicas de donantes y receptores y en aspectos técnicos de la procura e implante- no muestran diferencias clínicamente significativas en la función del injerto post-trasplante. Tampoco, en la evaluación histológica del daño por isquemia-reperfusión, en la evolución y complicaciones biliares post-operatorias, en la tasa de rechazo agudo, ni en la sobrevida al año de injertos y pacientes.
Importante es mencionar que inicialmente todos los hígados fueron perfundidos con UW (única solución disponible en el país); pese a lo anterior, la comparación entre ambas soluciones nos parece igualmente válida, tal como ha sido comunicada por otros autores ${ }^{20,21}$.

La mayor cantidad de HTK utilizada respecto a UW, puede ser explicada por las recomendaciones iniciales del proveedor y eventualmente por la menor viscosidad de HTK. Pese a lo anterior, los costos finales por donante según la solución de 
preservación empleada fueron significativamente menores al utilizar HTK, lo cual significó un ahorro de USD\$ 1.660 por donante con respecto a UW. Así, en un programa de TH en Chile que realice unos $30 \mathrm{TH}$ al año, esto se podría traducir en un significativo ahorro de recursos (aproximadamente USD\$ 49.800 al año). Otras series han obtenido buenos resultados en TH utilizando menores cantidades de $\mathrm{HTK}^{20,22}$, lo que permitiría economizar aún más recursos.

Las curvas de aminotransferasas y de BT posttrasplantes fueron similares a lo descrito en otros estudios $^{22} \mathrm{y}$ no constituyeron un factor relevante en la evolución clínica. No se observó diferencias en la incidencia de disfunciones relacionadas al injerto. La falla primaria y las disfunciones iniciales en general son multifactoriales, donde los principales elementos que se han asociado son: una adecuada cadena de frio, la calidad de la preservación del órgano, la calidad del donante y los tiempos de isquemia ${ }^{17}$. En nuestra serie, los tiempos de isquemia, el grado de esteatosis del injerto y la proporción de donantes de criterios extendidos, fueron similares en ambos grupos. De esta manera, no se observó asociación entre el tipo de solución utilizada y las disfunciones o fallas primarias de injerto.

Algunos estudios han evaluado la incidencia de complicaciones biliares al utilizar estas soluciones, encontrando que disminuyen con $\mathrm{HTK}^{22,23}$. Ellos han planteado que las diferencias de viscosidad entre ambas, explicarían en parte estas diferencias. La menor viscosidad de HTK, se traduce en una infusión más rápida, lo que permitiría colocar al injerto en frío precozmente. Por otra parte, considerando que la vía biliar es irrigada exclusivamente desde la arteria, aquellas soluciones que lleguen fácilmente al lecho capilar distal, tendrían una ventaja sobre el daño isquémico de ésta. Se ha observado que a temperaturas sobre $4^{\circ} \mathrm{C}$, UW puede producir cristales de adenosina, los que ocluirían las ramas distales arteriales que irrigan el árbol biliar ${ }^{24}$. Esto no ocurre con HTK, que puede ser conservada entre 4 y $15^{\circ} \mathrm{C}^{24}$. En cualquier caso, la reducción del TIF y un adecuado lavado de la vía biliar durante la procura, parecen disminuir la incidencia de complicaciones biliares ${ }^{24}$. En nuestra serie, el TIF fue discretamente menorcon HTK, lo que puede atribuirse en parte, a que los injertos perfundidos con UW provenían inicialmente de centros más alejados que aquellos perfundidos con HTK. Concordante con este mayor TIF, observamos que el número de complicaciones biliares fue algo más frecuente con UW, aunque esta diferencia no alcanzó a ser significativa.

Diversos estudios han señalado que el grado de infiltración de neutrófilos y necrosis de los hepatocitos en la biopsia post-reperfusión refleja el grado de daño por preservación ${ }^{25}$. Este último, se ha relacionado con la incidencia de disfunción inicial del injerto, así como con el desarrollo de complicaciones biliares $^{26}$. Si bien estos hallazgos no permiten la prevención de estas complicaciones, sí permiten anticipar y programar su estrategia terapéutica. En nuestra serie, los hallazgos histológicos en las biopsias post-reperfusión no mostraron diferencias entre ambos grupos, observándose en la mayoría de las biopsias ausencia de daño o daño leve, lo que fue concordante con la favorable evolución clínica.

Finalmente, la sobrevida de los injertos al año post-trasplante fue similar con ambas soluciones, no constituyendo el medio de preservación una variable asociada a sobrevida, aun posterior a su ajuste por confundentes. Publicaciones anteriores han demostrado sobrevidas similares en estudios aleatorizados de injertos preservados con ambas soluciones. Rayya y cols. ${ }^{21}$ publicaron una sobrevida de injerto de $78 \%$ con UW y de $71 \%$ con HTK a 12 mesespost-trasplante. En tanto Mangus y cols. ${ }^{27}$ observaron una sobrevida al año cercana a $80 \%$ con ambas soluciones.

En conclusión, la solución histidina-triptofano-ketoglutarato constituye una alternativa segura en la preservación de injertos hepáticos obtenidos de donantes fallecidos, ahorrando recursos significativamente en trasplante hepático.

\section{Referencias}

1. Kalayoglu M, Sollinger HW, Stratta R, D'Alessandro A, Hoffmann R, Pirsch J, et al. Extended preservation of the liver for clinical transplantation. Lancet 1988; 1 (8586): 617-9.

2. Todo S, Nery J, Yanaga K, Podesta L, Gordon RD, Starzl TE. Extended preservation of human liver grafts with UW solution. JAMA 1989; 261 (5): 711-4.

3. Belzer F, D`Alessandro A, Hoffman RM, Knechtle S, Reed A, Pirsch JD, et al. The use of UW solution in clinical transplantation. A 4-year experience. Ann Surg 1992; 215 (6): 579-83 
4. Bretschneider HJ. Myocardial protection. Thorac Cardiovasc Surg 1980; 28 (5): 295-302.

5. Menasché $P$, Termignon JL, Pradier F, Grousset C, Mouas C, Alberici G, et al. Experimental evaluation of Celsior, a new heart preservation solution. Eur J Cardiothorac Surg 1994; 8 (4): 207-13.

6. Gubernatis G, Pichlmayr R, Lamesch P, Grosse H, Bornscheuer A, Meyer HJ, et al. HTK-solution (Bretschneider) for human liver transplantation. First clinical experiences. Langenbecks Arch Chir 1990; 375 (2): 66-70.

7. Cavallari A, Cillo U, Nardo B, Filipponi F, Gringeri E, Montalti R, et al. A multi-center pilot prospective study comparing Celsior and University of Wisconsin preserving solutions for use in liver transplantation. Liver Transpl 2003; 9 (8): 814-21.

8. De Boer J, De Meester J, Smits JM, Groenewoud AF, Bok A, van der Velde O, et al. Eurotransplant randomized multicenter kidney graft preservation study comparing HTK with UW and Euro-Collins. Transpl Int 1999; 12 (6): 447-53.

9. Lamesch P, Raygrotzki S, Kherer G, Gubernatis G, Bretschneider HJ, Pichlmayr R. Preservation of the liver with the HTK solution. Transplant Proc 1990; 22 (2): 518-9.

10. Aminalai A, Kehrer G, Grossmann F, Richter J, Bretschneider HJ. Morphological investigation of the porcine liver directly following preservation with Euro-Collins, University of Wisconsin and Bretschneider's HTK solution. Langenbecks Arch Chir 1992; 377 (2): 81-8.

11. Feng XN, Xu X, Zheng SS. Current status and perspective of liver preservation solutions. Hepatobiliary Pancreat Dis Int 2006; 5 (4): 490-4.

12. De Ville de Goyet J, Hausleithner V, Malaise J, Reding $\mathrm{R}$, Lerut J, Jamart J, et al. Liver procurement without in situ portal perfusion. A safe procedure for more flexible multiple organ harvesting. Transplantation 1994; 57 (9): 1328-32.

13. Martínez J, Jarufe N, Crovari F, Álvarez S, Arrese M, Pérez RM, et al. Procura de hígado para trasplante hepático ortotópico. Comparación entre dos técnicas. Rev Chil Cir 2005; 57 (1): 26-32.

14. Merion RM, Goodrich NP, Feng S. How can we define expanded criteria for liver donors? Hepatology 2006; 45 (4): 484-8.

15. Martínez J. Implantación con técnica de Piggy-Back y anastomosis cavo-cava latero-lateral en trasplante hepático ortotópico. Experiencia inicial en un Centro Universitario. Rev Chil Cir 2004; 56 (4): 317-31.

16. Stockmann M, Lock JF, Malinowski M, Seehofer D, Puhl G, Pratschke J, et al. How to define initial poor graft function after liver transplantation? - a new functional definition by the LiMAx test. Transpl Int 2010; 23 (10): 1023-32.

17. Uemura T, Randall HB, Sánchez EQ, Ikegami T, Narasimhan G, McKenna GJ, et al. Liver retransplantation for primary nonfunction: analysis of a 20-year single-center experience. Liver Transpl 2007; 13 (2): 227-33.

18. Hepp J, Ríos H, Suárez L, Zaror M, Quiroga M, Rodríguez G, et al. Trasplante hepático en adultos: casuística de Clínica Alemana de Santiago. Rev Med Chile 2002; 130 (7): 779-86.

19. Buckel E, Silva G, Brahm J, Zacarías J, Ceresa S, Carvajal $\mathrm{C}$, et al. Experiencia de un centro en trasplante hepático en adultos y niños. Rev Med Chile 1996; 124 (1): 27-36.

20. Mangus RS, Tector AJ, Agarwal A, Vianna R, Murdock P, Fridell JA. Comparison of histidine-tryptophanketoglutarate solution (HTK) and University of Wisconsin solution (UW) in adult liver transplantation. Liver Transpl 2006; 12 (2): 226-30.

21. Rayya F, Harms J, Martin AP, Bartels M, Hauss J, Fangmann J. Comparison of histidine-tryptophan-ketoglutarate solution and University of Wisconsin solution in adult liver transplantation. Transplant Proc 2008; 40 (4): 891-94.

22. Agarwal A, Murdock P, Pescovitz MD, Goggins WC, Milgrom ML, Fridell JA. Follow-up experience using histidine-tryptophan ketoglutarate solution in clinical pancreas transplantation. Transplant Proc 2005; 37 (8): 3523-26.

23. Moench C, Otto G. Ischemic type biliary lesions in histidine-tryptophan ketoglutarate (HTK) preserved liver grafts. Int J Artif Organs 2006; 29 (3): 329-34.

24. Tullius S, Filatenkow A, Horch D, Mehlitz T, Selke AR, Pratschke J, et al. Accumulation of crystal deposits in abdominal organs following perfusion with defrosted University of Wisconsin solution. Am J Transplant 2002; 2 (7): 627-30.

25. Gaffey MJ, Boyd JC, Traweek ST, Ali A, Rezeig M, Caldwell SH, et al. Predictive value of intraoperative biopsies and liver function tests for preservation injury in orthotopic liver transplantation. Hepatology 1997; 25 (1): 184-9.

26. Busquets J, Figueras J, Serrano T, Torras J, Ramos E, Rafecas A, et al. Postreperfusion biopsies are useful in predicting complications after liver transplantation. Liver Transpl 2001; 7 (5): 432-5.

27. Mangus RS, Fridell JA, Vianna RM, Milgrom MA, Chestovich P, Chihara RK, et al. Comparison of histidinetryptophan-ketoglutarate solution and University of Wisconsin solution in extended criteria liver donors. Liver Transpl 2008; 14 (3): 365-73. 